\title{
A novel somatic MAPK1 mutation in primary ovarian mixed germ cell tumors
}

\author{
YANG ZOU $^{1,2^{*}}$, WEI DENG ${ }^{1 *}$, FENG WANG $^{1,2}$, XIAO-HONG YU ${ }^{3}$, FA-YING LIU ${ }^{1,2}$, BI-CHENG YANG ${ }^{1}$, \\ MEI-ZHEN HUANG ${ }^{1,2}$, JIU-BAI GUO ${ }^{1,4,5}$, QIU-HUA XIE ${ }^{1,2,5}$, MING HE ${ }^{6}$ and OU-PING HUANG ${ }^{1,4}$ \\ ${ }^{1}$ Key Laboratory of Women's Reproductive Health of Jiangxi, ${ }^{2}$ Central Laboratory, \\ Departments of ${ }^{3}$ Pathology and ${ }^{4}$ Gynecology, Jiangxi Provincial Maternal and Child Health Hospital, \\ Nanchang, Jiangxi 330006; ${ }^{5}$ Graduate School of Nanchang University, Nanchang, Jiangxi 330031; \\ ${ }^{6}$ Department of Pharmacology and Molecular Therapeutics, Nanchang University, Nanchang, Jiangxi 330006, P.R. China
}

Received July 18, 2015; Accepted September 8, 2015

DOI: 10.3892/or.2015.4402

\begin{abstract}
A recent exome-sequencing study revealed prevalent mitogen-activated protein kinase 1 (MAPK1) p.E322K mutation in cervical carcinoma. It remains largely unknown whether ovarian carcinomas also harbor MAPK1 mutations. As paralogous gene mutations co-occur frequently in human malignancies, we analyzed here a total of 263 ovarian carcinomas for the presence of MAPK1 and paralogous MAPK3 mutations by DNA sequencing. A previously unreported MAPK1 p.D321N somatic mutation was identified in 2 out of $18(11.1 \%)$ ovarian mixed germ cell tumors, while no other MAPK1 or MAPK3 mutation was detected in our samples. Of note, OCC-115, the MAPK1-mutated sample with bilateral cancerous ovaries affected, harbored MAPK1 mutation in the right ovary while retained the left ovary intact, implicating that the genetic alterations underlying ovarian mixed germ cell tumor may be different, even in patients with similar genetic backgrounds and tumor microenvironments. The results of evolutionary conservation and protein structure modeling analysis implicated that MAPK1 p.D321N mutation may be pathogenic. Additionally, mutations in protein phosphatase 2 regulatory subunit $\alpha$ (PPP2R1A), ring finger protein 43 (RNF43), DNA directed polymerase $\varepsilon$ (POLE1), ribonuclease type III (DICER1), CCCTC-binding factor (CTCF),
\end{abstract}

Correspondence to: Professor Ming He, Department of Pharmacology and Molecular Therapeutics, Nanchang University, 461 Bayi Avenue, Nanchang, Jiangxi 330006, P.R. China

E-mail: jxhm56@hotmail.com

Professor Ou-Ping Huang, Key Laboratory of Women's Reproductive Health of Jiangxi, Jiangxi Provincial Maternal and Child Health Hospital, 318 Bayi Avenue, Nanchang, Jiangxi 330006, P.R. China E-mail: jxfbhop@126.com

*Contributed equally

Key words: MAPK1, MAPK3, mutation, ovarian mixed germ cell tumor, China ribosomal protein L22 (RPL22), DNA methyltransferase $3 \alpha$ (DNMT3A), transformation/transcription domain-associated protein (TRRAP), isocitrate dehydrogenase (IDH)1 and IDH2 were not detected in ovarian mixed germ cell tumors, implicating these genetic alterations may be not associated with MAPK1 mutation in the development of this malignancy. The present study identified a previously unreported MAPK1 mutation in ovarian mixed germ cell tumors for the first time, and this mutation may be actively involved in the tumorigenesis of this disease.

\section{Introduction}

Ovarian carcinoma is a leading cause of gynecologic malignancies (1). It is estimated that 5-year survival rates could be more than $90 \%$ in ovarian cancers experiencing early detection of the malignancy; nevertheless, due to the anatomical location the primary ovarian cancer is insidious, fewer than $20 \%$ of ovarian cancers could be detected at their early stages, this is also the main reason for the high lethality of this malignancy $(2,3)$. On the other hand, ovarian carcinoma is comprised of histologically diverse subtypes but still commonly treated as a single disorder with limited stratification based on histological characteristics or molecular genetic alterations, the 5-year survival rates of this malignancy remains under $50 \%(4,5)$. This prompted us to seek better understanding of the molecular genetic alterations contributing to the initiation and progression of ovarian cancer.

RAS/RAF/MEK/ERK cascade is a key signaling pathway regulating diverse biological processes such as cell proliferation, survival and programmed cell death $(6,7)$. Multiple lines of evidence have suggested that this signaling pathway is frequently deregulated in human cancers as a result of either genetic aberrations of their components or over-activation of upstream cell-surface receptors $(7,8)$. Amongst these reported genetic alterations, mutations in the BRAF and three RAS members (KRAS, NRAS and HRAS) were prevalent in human cancers, while mutations in other components such as MAPK1 (ERK2) were relatively infrequently detected (9-12). Of note, a large-scale sequencing study have identified a high frequency of MAPK1 mutations $(6 / 79,7.6 \%)$ in primary 
cervical squamous cell carcinomas, among the 6 patients with MAPK1 mutations, 4 samples harbored MAPK1 p.E322K mutation and the remaining 2 samples harbored either MAPK1 p.E81K or p.E220K mutation; thus MAPK1 p.E322 mutation was considered as the potential mutational hot spot $(4 / 6$, $66.7 \%$ ) in cervical cancer (13). Till now, the mutational statues of MAPK1 mutations in other cancer types, such as ovarian cancer, remain largely unexplored.

Considering the fact that there existed some overlaps of molecular genetic aberrations between ovarian and cervical carcinomas, such as prevalent PIK3CA and TP53 mutations $(13,14)$, it raises the possibility that ovarian carcinoma may also harbor MAPK1 mutations. On the other hand, homologous residues of paralogous genes are frequently mutated in certain cancer types, thus we wanted to explore the possibility that MAPK3 p.E339 residue, the homologue residue of MAPK1 p.E322, would be also mutated in ovarian carcinoma.

In the present study, we analyzed a cohort of 263 Chinese ovarian cancer samples with distinct subtypes for the presence of MAPK1 and MAPK3 mutations. Furthermore, the potential hotspot mutations in several newly-identified cancer-related genes were also analyzed in our samples previously and here, including PPP2R1A, RNF43, POLE1, DICER1, CTCF, RPL22, DNMT3A, TRRAP, IDH1 and IDH2 (15-18), with the aim of exploring the possibility that these mutations would play synergistic role with MAPK1 mutation in the development of this malignancy.

\section{Materials and methods}

Patients and ethics statement. A collection of 263 formalin-fixed, paraffin-embedded (FFPE) samples with distinct subtypes of ovarian carcinomas were recruited from the archives of Department of Pathology, Jiangxi Provincial Maternal and Child Health Hospital from March 2007 to May 2015. Each sample was reviewed by two experienced pathologists, all of the recruited samples contained $>70 \%$ cancerous cells and did not undergo chemotherapy and/or radiotherapy. Among these patients, 251 patients were described previously (15-18) and the additional 12 patients diagnosed with ovarian mixed germ cell tumor were newly recruited (Table I). In addition, among these cases, 238 paired adjacent non-cancerous samples were also taken from archival blocks of these oophorectomy samples where no cancerous cells were identified by hematoxylin-eosin staining. The Institutional Review Board of Jiangxi Provincial Maternal and Child Health Hospital approved this study and an informed consent was obtained from each patient prior to this study. The study was conducted according to the Declaration of Helsinki.

MAPK1 and MAPK3 hotspot mutation analyses. The genomic DNA of each sample was isolated by commercially available kits (OMEGA Bio-Tek Inc., Doraville, GA, USA) and the quantity and quality of DNA was determined spectrophotometrically. For sequence analysis of the potential MAPK1 and MAPK3 mutations, a 246- and a 231-bp PCR fragment covering MAPK1 p.E322 and the paralogous MAPK3 p.E339 residue was amplified by PCR, respectively, with the following primer pairs:
Table I. The mutations of the MAPK1 and MAPK3 genes in 263 samples with distinct subtypes of ovarian tumors.

\begin{tabular}{lccc}
\hline Subtype & No. & $\begin{array}{c}\text { MAPK1 } \\
\text { p.D321N }\end{array}$ & $\begin{array}{c}\text { MAPK3 } \\
\text { p.E339 }\end{array}$ \\
\hline Epithelial & & & \\
Serous & 76 & $0 / 76$ & $0 / 76$ \\
Clear cell & 43 & $0 / 43$ & $0 / 43$ \\
Endometrioid & 37 & $0 / 37$ & $0 / 37$ \\
Mucinous & 15 & $0 / 15$ & $0 / 15$ \\
Undifferentiated & 3 & $0 / 3$ & $0 / 3$ \\
Unclassified & 4 & $0 / 4$ & $0 / 4$ \\
Transitional cell & 3 & $0 / 3$ & $0 / 3$ \\
Mixed & 2 & $0 / 2$ & $0 / 2$ \\
Non-epithelial & & & \\
germ cell tumor & & & \\
Yolk sac & 11 & $0 / 11$ & $0 / 11$ \\
Dysgerminoma & 7 & $0 / 7$ & $0 / 7$ \\
Teratoma & 9 & $0 / 9$ & $0 / 9$ \\
Mixed & 18 & $2 / 18$ & $0 / 18$ \\
Gender cord-stromal & & & \\
Granulosa cell & 16 & $0 / 16$ & $0 / 16$ \\
Sertoli-Leydig & 2 & $0 / 2$ & $0 / 2$ \\
Krukenberg tumor & 17 & $0 / 17$ & $0 / 17$ \\
\hline
\end{tabular}

forward, 5'-CTGCTCTCACTACTGCAAAACC-3' and reverse, 5'-TGGCAGCAGGTATATCTCAGG-3' for MAPK1; forward, 5'-CTGACTCCTGCCCTTCCATA-3' and reverse, 5'-GGGTGGTAGAGACAGCAAGG-3' for MAPK3. A total of $200 \mathrm{ng}$ of genomic DNA was used for each amplification reaction in a total volume of $30 \mu \mathrm{l}$, after an initial denaturation step at $94^{\circ} \mathrm{C}$ for $3 \mathrm{~min}, 35$ cycles were run with the following conditions: denaturation at $94^{\circ} \mathrm{C}$ for $30 \mathrm{sec}$, annealing at 55 or $60^{\circ} \mathrm{C}$ for $30 \mathrm{sec}$, extension at $72^{\circ} \mathrm{C}$ for $30 \mathrm{sec}$; finally followed by a final extension at $72^{\circ} \mathrm{C}$ for $10 \mathrm{~min}$. All PCR reactions were performed in a Thermal Cycler 2720 (Applied Biosystems, Foster City, CA, USA). After purification, the PCR products were subjected to DNA sequencing on an ABI Prism 3730 DNA sequencer (Applied Biosystems). An independent PCR and bidirectional sequencing was used to verify the identified mutations. The somatic status of these identified MAPK1 mutations were determined by sequencing the MAPK1 gene in correspondingly paired adjacent normal tissues.

PPP2R1A,RNF43, POLE1,DICER1,CTCF, RPL22,DNMT3A, TRRAP, IDH1 and IDH2 hotspot mutation analyses. The mutational status of several potential ovarian cancer-related genes were analyzed in our samples previously (15-18) and here (ovarian mixed germ cell tumor, $\mathrm{n}=12$ ), including PPP2R1A, RNF43, POLE1, DICER1, CTCF, RPL22, DNMT3A, TRRAP, IDH1 and IDH2, the PCR and DNA sequencing reactions were performed as previously described (15-18).

Evolutionary conservation analysis. Twenty-six vertebrate species were selected from GenBank to analyze the 


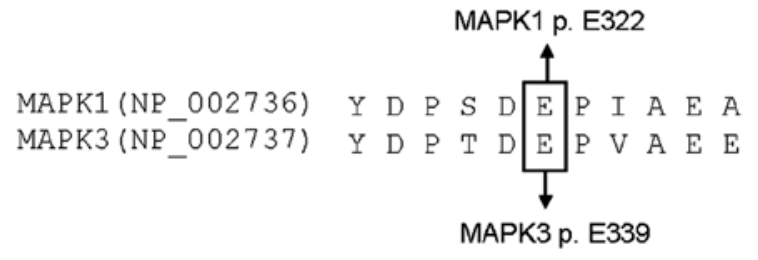

Figure 1. Protein sequence homology analysis of MAPK1 and MAPK3.

evolutionary conservation status of MAPK1 mutation, including Homo sapiens (NP_002736), Pan troglodytes (XP_003317171), Mus musculus (NP_036079), Rattus norvegicus (NP_446294), Cricetulus griseus (XP_007641645), Odobenus rosmarus divergens (XP_004400572), Oryctolagus cuniculus (XP_008270402), Tupaia chinensis (XP_006140283), Ovis aries (XP_0040177265), Canis lupus familiaris (NP_001104270), Sus scrofa (NP_001185851), Bos taurus (NP_786987), Vicugna pacos (XP_006213368), Equus caballus (XP_005612442), Alligator mississippiensis (XP_006269381), Chelonia mydas (XP_007054992), Lipotes vexillifer (XP_007451077), Xenopus laevis (NP_001083548), Python bivittatus (XP_007422500), Columba livia (XP_005515231), Zonotrichia albicollis (XP_005488954), Eptesicus fuscus (XP_0081408655), Gallus gallus (NP_989481), Astyanax mexicanus (XP_007229493), Poecilia reticulata (XP_008417574) and Danio rerio (NP_878308).

Protein structural modeling. The protein structural modeling was performed by DeepView Swiss-PdbViewer 4.0 software (19). Three PDB structures of human MAPK1 were available (3sa0.1.A, 1wzy.1.A and 4qte.1.A) in the ExPASy database (http://www.expasy.org). Based on these structures, by displaying 'show backbone oxygen', 'show dots surface' and 'sender in solid 3D', wild-type MAPK1 were built firstly and the p.D321N mutant MAPK1 was subsequently modeled by changing aspartic acid 321 to asparagine. Additionally, p.D321V mutant MAPK1 was also modeled according to the same procedure.

\section{Results}

MAPK1 and MAPK3 mutations in ovarian carcinoma. The clinical information of the sample cohort has been described previously (15-17). A total of 263 ovarian carcinomas were screened for the potential mutations in the MAPK1 p.E322 and paralogous MAPK3 p.E339 residues (Table I and Fig. 1). Although MAPK1 p.E322 mutations were not detected in these samples, intriguingly, a novel, previously unreported mutation in the 321 st residue [p.D321N, c. $(961 \mathrm{G}>\mathrm{A} ; 963$ $\mathrm{C}>\mathrm{T}$ )] adjacent to MAPK1 p.E322, was detected in 2 out of $18(11.1 \%)$ samples with ovarian mixed germ cell tumor, and the somatic status of these mutation was confirmed by sequencing the correspondingly adjacent normal tissues (Table I and Fig. 2A). In addition, no mutations were detected in the remaining samples (Table I). The age of the patients with mutations (OCC-44 and OCC-115) was 28 and 30 years, and affected the right and bilateral ovaries, respectively; while the 16 ovarian mixed germ cell tumors with wild-type MAPK1 affected 8 left, 3 bilateral and 5 right ovaries, respectively (17). Of note, OCC-115, the sample with bilateral ovaries affected, whose bilateral cancerous ovaries were obtained and MAPK1 p.D321N mutation was detected only in the right ovary while absent in the left cancerous ovary. Moreover, no MAPK3 mutation was detected in our samples (Table I and Fig. 2B), including the MAPK3 p.D338 and p.E339 residues, which were the corresponding paralogous residues of MAPK1 p.D321 and p.E322, respectively.

Association of MAPK1 mutation with other genetic alterations in ovarian mixed germ cell tumor. We screened our samples for the presence of PPP2R1A, RNF43, POLE1, DICER1, CTCF, RPL22, DNMT3A, TRRAP, IDH1 and IDH2 mutations in our prior (15-18) and the present study. Nevertheless, no mutation was detected in these genes.

Evolutionary conservation analysis and protein structural modeling. MAPK1 p.D321 residue is located in the cytoplasmic retention motif (http://www.uniprot.org/) and the result of evolutionary conservation analysis suggested that this residue was highly conserved in vertebrate from Homo sapiens to Danio rerio (Fig. 3). Protein structural modeling results suggested that the MAPK1 p.D321N mutant and another p.D321 mutant (p.D321V) which was identified in malignant melanomas (20), exhibited structural changes in all of the 3 PDB versions of MAPK1 protein. Herein, one of the PDB structures (3sa0.1.A) used in the present study is displayed (Fig. 4).

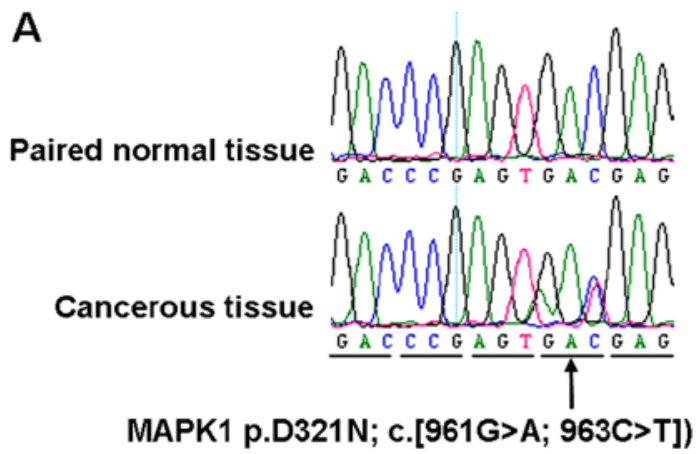

B

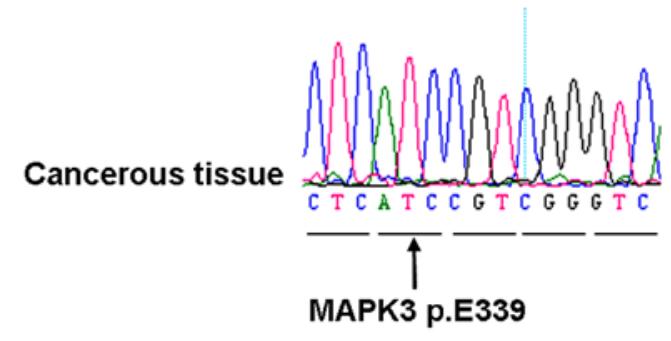

Figure 2. Representative sequencing electropherograms of MAPK1, the arrow refers to locations of the mutation (A). A representative sequencing electropherograms of MAPK3 (B). 


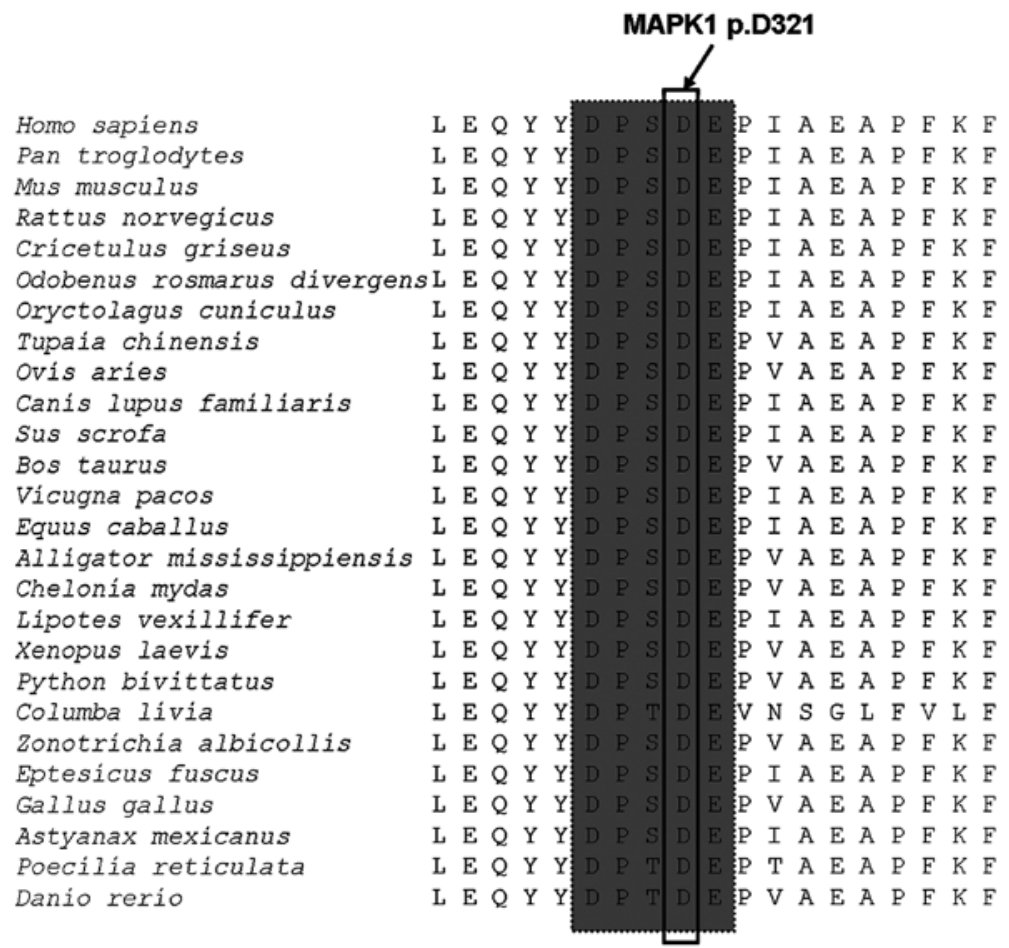

Figure 3. Results of the evolutionary conservation analysis of MAPK1 p.D321 residue in 26 vertebrate species and the cytoplasmic retention motif of MAPK1 (marked in grey color).

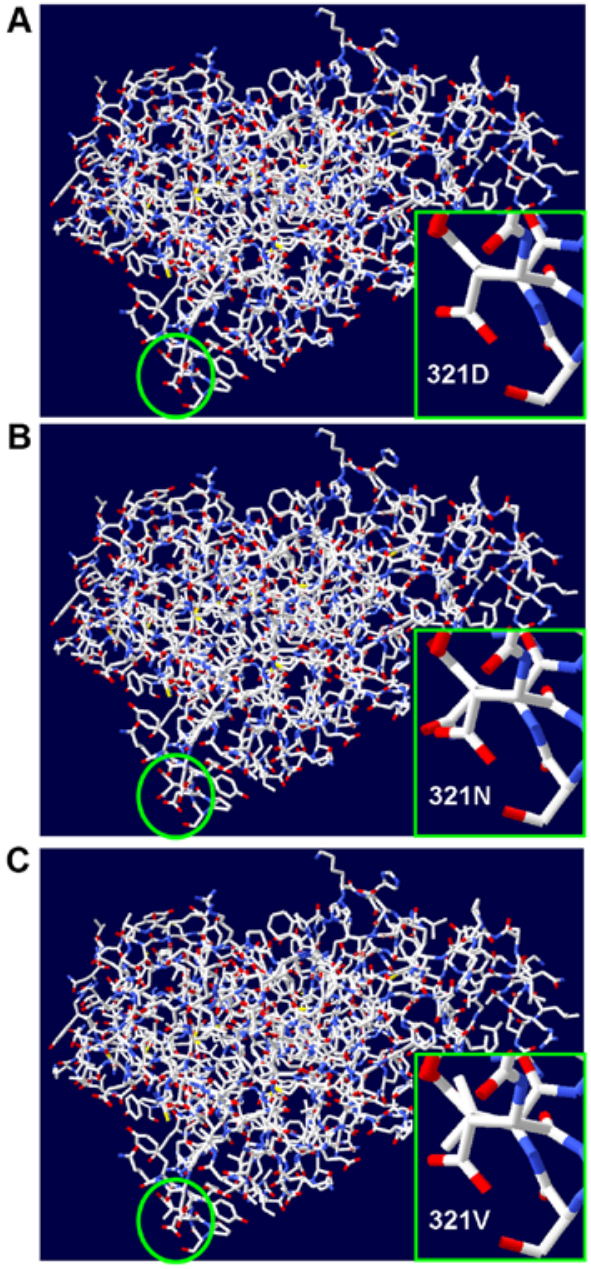

Figure 4. Protein structural modeling of MAPK1 p.D321 wild-type (A), MAPK1 p.D321N (B) and p.D321V (C) mutants.

\section{Discussion}

Previous studies have suggested that MAPK1 mutations were either rare or absent in human cancers (21-23) (http://www. sanger.ac.uk/cosmic). However, a recent integrated genomic characterization study identified a high frequency of MAPK1 mutations in primary cervical squamous cell carcinomas (13).

Ovarian mixed germ cell tumor is an ovarian germ cell tumor containing two or more types of germ cell components with extremely low incidence worldwide (24). Up to date, the detailed molecular aberrations underlying ovarian mixed germ cell tumor remains largely unknown. In the present study, a novel MAPK1 p.D321N somatic mutation was detected in 2 out of 18 ovarian mixed germ cell tumors but not in other subtypes of ovarian carcinoma, implying this mutation may play an active role specifically in the pathogenesis of ovarian mixed germ cell tumor. Intriguingly, OCC-115, the MAPK1-mutated sample with both ovaries affected, harbored MAPK1 mutation in the right ovary only but not in the contralateral cancerous ovary. The discrepant mutational status of MAPK1 between the paired cancerous ovaries in the same patient indicated that the genetic alterations underlying ovarian mixed germ cell tumor may be more complicated than we thought, even in samples with similar genetic backgrounds and tumor microenvironments. To our knowledge, this is the first report revealing a novel MAPK1 mutation in ovarian mixed germ cell tumor, and that this mutation may be actively involved in the tumorigenesis process and may be a potential molecular therapy target for this disorder.

Multiple genetic alterations are necessary for the development of human cancers, it thus would be crucial for the diagnosis and therapy of cancer patients to understand their underlying combined events of genetic alterations $(13,14,22)$. 
Nevertheless, we failed to detect any mutations in the 10 novel cancer-related genes in our ovarian mixed germ cell tumors previously (15-18) and here, including PPP2R1A, RNF43, POLE1, DICER1, CTCF, RPL22, DNMT3A, TRRAP, IDH1 and IDH2. There results implicated that these genetic alterations may not play synergistic roles with MAPK1 p.D321N mutation in the development of the ovarian mixed germ cell tumor. In addition, no MAPK3 mutation was detected in our sample cohort, indicating that MAPK3 mutations may be not actively involved in the pathogenesis of ovarian carcinoma.

In contrast to the mixed subtype, MAPK1 mutations were not detected in other subtypes of germ cell tumors, including patients with yolk sac $(n=11)$, dysgerminoma $(n=7)$ and teratoma $(n=9)$ subtypes. The inconsistent mutational status of MAPK1 in various subtypes of germ cell tumor further indicated that germ cell tumor of ovary was heterogeneous and the potential molecular genetic alterations underlying these tumor subtypes may be quite complex (25).

In addition, MAPK1 mutations were not found in the 76 ovarian serous or 15 mucinous carcinomas. This was consistent with several previous observations based on whole-exome or genome sequencing projects, where MAPK1 mutations were absent in 32 small cell (26), 15 mucinous (27), 2 serous borderline (28) and 997 serous subtypes of the ovary (14) (http://www.sanger.ac.uk/cosmic). Together, these combined data show that MAPK1 mutations may be rare in ovarian serous or mucinous carcinomas. Similarly, MAPK1 mutations were not detected in other primary and secondary ovarian carcinomas in our samples, implying that MAPK1 mutations may not be involved in tumorigenesis of these tumor subtypes.

Functionally, it is not yet clear whether MAPK1 p.D321N mutation would play substantial role in the pathogenesis of ovarian mixed germ cell tumor. Prior large-scale sequencing efforts have detected MAPK1 p.D321N (c.961G>A) mutation in 1 out of $35(2.9 \%)$ patients with head and neck squamous cell carcinoma (29) (http://www.sanger.ac.uk/cosmic), and MAPK1 p.D321V (c.962A >T) mutation in 1 out of 147 malignant melanomas (20). In addition, the results of protein sequence homology and evolutionary conservation analyses indicated that MAPK1 p.D321 residue located in the cytoplasmic retention motif (http://www.uniprot.org/) and was highly conserved in 26 vertebrate species from Homo sapiens to Danio rerio. On the other hand, the results of protein structure modeling indicated that the mutations in the MAPK1 p.D321 residue (p.D321N and p.D321V) would lead to protein structure changes and may affect the activity of MAPK1. Altogether, we speculated that MAPK1 p.D321N mutation may promote the development of ovarian mixed germ cell tumor, via change of MAPK1 activity or subcellular localization. However, further functional assays would be necessary to confirm these speculations.

A main limitation of the present study was that we have screened only a short DNA fragment spanning the potential mutational hotspot of MAPK1 and MAPK3, it could be more informative to detect the entire coding region of the MAPK1 and MAPK3 genes. However, this is limited mainly by our available DNA materials isolated from FFPE tissues and we thus failed to test this aspect. Furthermore, we have analyzed the mutational status of MAPK1 in a total of 18 ovarian mixed germ cell tumors, further analysis using larger sample sizes will be helpful for validating the accurate frequency of MAPK1 p.D321N mutation in this specific subtype of ovarian carcinoma.

In conclusion, our study reveals a novel mutation associated with ovarian mixed germ cell tumor but not other subtypes, thus, this mutation may play active role in the progression of ovarian mixed germ cell tumor and may be a promising therapeutic target for this disorder. Moreover, absence of MAPK3, PPP2R1A, RNF43, POLE1, DICER1, CTCF, RPL22, DNMT3A, TRRAP, IDH1 and IDH2 mutations in MAPK1-mutated ovarian mixed germ cell tumors indicated that these mutations may not cooperate with MAPK1 mutation in the development of this disorder.

\section{Acknowledgements}

We thank all participants involved in the present study. The present study was supported by grants from the National Natural Science Foundation of China (no. 81260384), the Natural Science Foundation of Jiangxi (no. 20142BAB215003) and Basic Equipment Grant for Research Institute of Jiangxi (no. 20151BBA13051).

\section{References}

1. Jemal A, Bray F, Center MM, Ferlay J, Ward E and Forman D: Global cancer statistics. CA Cancer J Clin 61: 69-90, 2011.

2. Munkarah A, Chatterjee M and Tainsky MA: Update on ovarian cancer screening. Curr Opin Obstet Gynecol 19: 22-26, 2007.

3. Tinelli A, Vergara D, Martignago R, Leo G, Pisanò $M$ and Malvasi A: An outlook on ovarian cancer and borderline ovarian tumors: Focus on genomic and proteomic findings. Curr Genomics 10: 240-249, 2009.

4. Lowe KA, Chia VM, Taylor A, O'Malley C, Kelsh M, Mohamed M, Mowat FS and Goff B: An international assessment of ovarian cancer incidence and mortality. Gynecol Oncol 130: 107-114, 2013.

5. Davis A, Tinker AV and Friedlander M: 'Platinum resistant' ovarian cancer: What is it, who to treat and how to measure benefit? Gynecol Oncol 133: 624-631, 2014.

6. De Luca A, Maiello MR, D’Alessio A, Pergameno M and Normanno N: The RAS/RAF/MEK/ERK and the PI3K/AKT signalling pathways: Role in cancer pathogenesis and implications for therapeutic approaches. Expert Opin Ther Targets 16 (Suppl 2): S17-S27, 2012.

7. Steelman LS, Franklin RA, Abrams SL, Chappell W, Kempf CR, Bäsecke J, Stivala F, Donia M, Fagone P, Nicoletti F, et al: Roles of the Ras/Raf/MEK/ERK pathway in leukemia therapy. Leukemia 25: 1080-1094, 2011.

8. McCubrey JA, Steelman LS, Chappell WH, Abrams SL, Wong EW, Chang F, Lehmann B, Terrian DM, Milella M, Tafuri A, et al: Roles of the Raf/MEK/ERK pathway in cell growth, malignant transformation and drug resistance. Biochim Biophys Acta 1773: 1263-1284, 2007.

9. Holderfield M, Deuker MM, McCormick F and McMahon M: Targeting RAF kinases for cancer therapy: BRAF-mutated melanoma and beyond. Nat Rev Cancer 14: 455-467, 2014.

10. Wang Y, Kaiser CE, Frett B and Li HY: Targeting mutant KRAS for anticancer therapeutics: A review of novel small molecule modulators. J Med Chem 56: 5219-5230, 2013.

11. Tsai JH, Huang WC, Jhuang JY, Jeng YM, Cheng ML, Chiu HY, Kuo KT and Liau JY: Frequent activating HRAS mutations in trichilemmoma. Br J Dermatol 171: 1073-1077, 2014.

12. Trietsch MD, Spaans VM, ter Haar NT, Osse EM, Peters AA, Gaarenstroom KN and Fleuren GJ: CDKN2A(p16) and HRAS are frequently mutated in vulvar squamous cell carcinoma. Gynecol Oncol 135: 149-155, 2014. 
13. Ojesina AI, Lichtenstein L, Freeman SS, Pedamallu CS, Imaz-Rosshandler I, Pugh TJ, Cherniack AD, Ambrogio L, Cibulskis K, Bertelsen B, et al: Landscape of genomic alterations in cervical carcinomas. Nature 506: 371-375, 2014.

14. Bell DBA, Birrer M, Chien J, Cramer D, Dao F, Dhir R, DiSaia P, Gabra H, Glenn P, Godwin A, et al; Cancer Genome Atlas Research Network: Integrated genomic analyses of ovarian carcinoma. Nature 474: 609-615, 2011.

15. Wang F, Zou Y, Liu FY, Yu XH, Huang H, Zhang N, Qi YY, Liu RF, Liu XY, Chen J, et al: Infrequent mutations of the PPP2R1A and PPP2R1B genes in patients with ovarian cancer. Mol Med Rep 7: 1826-1830, 2013.

16. Zou Y, Wang F, Liu FY, Huang MZ, Li W, Yuan XQ, Huang OP and He M: RNF43 mutations are recurrent in Chinese patients with mucinous ovarian carcinoma but absent in other subtypes of ovarian cancer. Gene 531: 112-116, 2013.

17. Zou Y, Liu FY, Liu H, Wang F, Li W, Huang MZ, Huang Y, Yuan XQ, Xu XY, Huang OP, et al: Frequent POLE1 p.S297F mutation in Chinese patients with ovarian endometrioid carcinoma. Mutat Res 761: 49-52, 2014.

18. Zou Y, Huang MZ, Liu FY, Yang BC, Wang LQ, Wang F, Yu XH, Wan L, Wan XD, Xu XY, et al: Absence of DICER1, CTCF, RPL22, DNMT3A, TRRAP, IDH1 and IDH2 hotspot mutations in patients with various subtypes of ovarian carcinomas. Biomed Rep 3: 33-37, 2015.

19. Guex N and Peitsch MC: SWISS-MODEL and the SwissPdbViewer: An environment for comparative protein modeling. Electrophoresis 18: 2714-2723, 1997.

20. Krauthammer M, Kong Y, Ha BH, Evans P, Bacchiocchi A McCusker JP, Cheng E, Davis MJ, Goh G, Choi M, et al: Exome sequencing identifies recurrent somatic RAC1 mutations in melanoma. Nat Genet 44: 1006-1014, 2012.

21. Muzny DMBM, Chang K, Dinh HH, Drummond JA, Fowler G, Kovar CL, Lewis LR, Morgan MB, Newsham IF, Reid JG, et al; Cancer Genome Atlas Network: Comprehensive molecular characterization of human colon and rectal cancer. Nature 487: $330-337,2012$
22. Biankin AV, Waddell N, Kassahn KS, Gingras MC, Muthuswamy LB, Johns AL, Miller DK, Wilson PJ, Patch AM, Wu J, et al; Australian Pancreatic Cancer Genome Initiative: Pancreatic cancer genomes reveal aberrations in axon guidance pathway genes. Nature 491: 399-405, 2012.

23. Landau DA, Carter SL, Stojanov P, McKenna A, Stevenson K, Lawrence MS, Sougnez C, Stewart C, Sivachenko A, Wang L, et al: Evolution and impact of subclonal mutations in chronic lymphocytic leukemia. Cell 152: 714-726, 2013.

24. Goyal LD, Kaur S and Kawatra K: Malignant mixed germ cell tumour of ovary - an unusual combination and review of literature. J Ovarian Res 7: 91, 2014.

25. Heravi-Moussavi A, Anglesio MS, Cheng SW, Senz J, Yang W, Prentice L, Fejes AP, Chow C, Tone A, Kalloger SE, et al: Recurrent somatic DICER1 mutations in nonepithelial ovarian cancers. N Engl J Med 366: 234-242, 2012.

26. Witkowski L, Carrot-Zhang J, Albrecht S, Fahiminiya S, Hamel N, Tomiak E, Grynspan D, Saloustros E, Nadaf J, Rivera B, et al: Germline and somatic SMARCA4 mutations characterize small cell carcinoma of the ovary, hypercalcemic type. Nat Genet 46: 438-443, 2014.

27. Ryland GL, Hunter SM, Doyle MA, Rowley SM, Christie M, Allan PE, Bowtell DD, Gorringe KL and Campbell IG; Australian Ovarian Cancer Study Group: RNF43 is a tumour suppressor gene mutated in mucinous tumours of the ovary. J Pathol 229: 469-476, 2013.

28. Boyd J, Luo B, Peri S, Wirchansky B, Hughes L, Forsythe C and $\mathrm{Wu} \mathrm{H}$ : Whole exome sequence analysis of serous borderline tumors of the ovary. Gynecol Oncol 130: 560-564, 2013.

29. Pickering CR, Zhang J, Yoo SY, Bengtsson L, Moorthy S, Neskey DM, Zhao M, Ortega Alves MV, Chang K, Drummond J, et al: Integrative genomic characterization of oral squamous cell carcinoma identifies frequent somatic drivers. Cancer Discov 3: 770-781, 2013. 